\title{
Non-serious Adverse Event
}

National Cancer Institute

\section{Source}

National Cancer Institute. Non-serious Adverse Event. NCI Thesaurus. Code C41336.

Any adverse experience that does not meet the definition of serious. Non-serious adverse events are classified as mild, moderate and severe. 